Original Paper http://ajol.info/index.php/ijbcs http://indexmedicus.afro.who.int

\title{
Valorisation des produits laitiers dans les ménages Peul du Nord-Est du Bénin
}

\author{
Roukayath CHABI TOKO ${ }^{1,2 *}$, Anselme ADEGBIDI ${ }^{2}$ et Philippe LEBAILLY ${ }^{1}$ \\ ${ }^{1}$ Université de Liège - Gembloux Agro-Bio Tech, Unité d'Économie et de Développement rural, Belgique. \\ ${ }^{2}$ Université d'Abomey-Calavi, Faculté des Sciences Agronomiques, Département d'Economie, Socio- \\ Anthropologie et Communication, Benin. \\ *Auteur correspondant ; E-mail : mahoussirouk@yahoo.fr; Tél: (00229) 95840204
}

\section{REMERCIEMENTS}

Les auteurs tiennent à exprimer leur gratitude à la Coopération Technique Belge (CTB) pour avoir financé cette recherche par l'octroi de la bourse de doctorat mixte.

\section{RÉSUMÉ}

La population pastorale et agro-pastorale du Bénin détient presque la totalité du cheptel bovin national. Le lait constitue le produit le plus fréquent issu de l'élevage bovin. Une enquête a été menée dans la commune de Kalalé au sein de 16 ménages peuls afin de comprendre l'organisation de ces ménages dans la gestion du lait. Il ressort que l'organisation autour du capital lait est genre sensible: l'homme aussi bien que la femme, dans leurs rôles respectifs, contribuent à assurer la pérennité du capital bétail. Le lait est géré de façon à privilégier la consommation du ménage et à assurer la reproduction du troupeau. La rémunération du travail fournit ainsi que l'équité dans la distribution des richesses au sein du ménage peul octroi aux femmes qui sont dans le troupeau plus de bénéfices issues du lait provenant du troupeau familial. La disponibilité des produits laitiers dépend de la saison et du milieu. Le lait prévu pour la consommation humaine est gérée de sorte à avoir, une utilisation optimale du lait et de la viande, afin de combler les besoins actuels des membres du ménage, d'assurer la reproduction des biens du ménage, avant d'alimenter le marché de produits laitiers.

(C) 2015 International Formulae Group. All rights reserved.

Mots clés: Contrôle du lait, gestion du lait, Peuls, produits laitiers.

\section{Dairy products valorization in Fulani households of North Eastern Benin}

\begin{abstract}
Pastoral and agro pastoral population of Benin holds almost all the national cattle herd. Milk is the most commonly product derived from cattle and its sale provides cash income to Fulani woman. A survey was conducted in Kalalé district within sixteen Fulani households to understand their milk management's organization. The organization around milk capital is gender sensitive. Man as well as woman, in their respective roles, help to ensure sustainability of livestock capital. Milk is managed to favor household consumption and ensure the herd's reproduction. Labor compensation and equity in wealth distribution within Fulani household granted to women who are in the herd more profits from milk of the family herd. Dairy products availability depends on the season and the area. The expected milk for human consumption is
\end{abstract}


managed so as to have an optimal use of milk and meat in order to meet the current needs of household members, to ensure the household goods' reproduction before supplying dairy market.

(C) 2015 International Formulae Group. All rights reserved.

Keywords: Milk control, milk management, Fulani, dairy Products.

\section{INTRODUCTION}

L'élevage domestique de subsistance est largement répandu dans l'économie béninoise et concerne toutes les populations rurales. Les bovins, et une portion de la production d'ovins et de caprins sont généralement associés aux pasteurs peuls qui détiennent approximativement $95 \%$ du cheptel national et qui pratiquent majoritairement l'élevage bovin (Somda et al., 2004; Youssao et al., 2013). Les Peuls du Bénin se sont sédentarisés dans des villages et des campements où ils cultivent les produits vivriers et élèvent le bétail (Jones, 1998). Les Peuls vivent dans des exploitations regroupées en hameaux. Leurs exploitations ont un fort degré de flexibilité et d'autonomie à travers une caractéristique association d'animaux, d'agriculture et d'activités de cueillette. C'est une économie familiale agro-pastorale qui caractérise donc la manière de vivre de la plupart des éleveurs peuls du Bénin (Bierschenk, 1998; Bierschenk et Forster, 2004). Dans ce système de production, l'élevage bovin joue un important rôle en fournissant des animaux sur pied, le lait pour la consommation du ménage et pour la commercialisation, et la fumure utilisée pour fertiliser les champs. Ce sont les produits laitiers commercialisés par ces femmes peules qui sont disponibles (Bierschenk et Forster, 2004), pour la majorité de la population sur les marchés du pays.

Selon l'Organisation des Nations Unies pour l'Alimentation et l'Agriculture (FAO), ce sont les petits producteurs qui produisent la grande majorité (entre 80 et $90 \%$ ) du lait, dans les pays en développement (FAO, 2015 a). C'est dans les pays en développement que la demande de produits laitiers connaîtra une augmentation où elle devrait progresser au rythme de 2,5\% par an; un chiffre comparable à celui des années 90 (FAO, 2004). Mais, les produits laitiers essentiellement importés sous forme de poudre entrent en compétition avec les produits laitiers pastoraux, comme dans le cas du Nigéria (Majekodunmi et al., 2014). Cependant, au Bénin, où le lait est également importé sous différentes formes (41 705 milliers de tonnes d'équivalent lait en 2013) (FAO, 2015b), la demande en produits laitiers pastoraux sur le terrain ne faibli pas, même si des statistiques restent inexistantes dans le domaine. La diversité des produits laitiers pastoraux, de même que leur spécificité, comparée aux produits laitiers importés, les rend plus attractifs pour la population; le cas du fromage peul qui est le produit laitier le plus répandu et le plus consommé au Bénin (Aïssi et al., 2009). Ce qui fait que le marché des produits laitiers pastoraux alimenté par les femmes peules, permet aux populations aussi bien rurales, qu'urbaine, de bénéficier de produits, comme le fromage traditionnel, dont les méthodes de conservation sont à la portée de tous les groupes socioculturels (Sessou et al., 2013).

Cependant, on constate facilement sur les marchés du pays que la demande en produits laitiers pastoraux est partiellement satisfaite en fonction des périodes dans l'année et des localités. Pour comprendre ce qui se passe et ainsi contribuer à l'amélioration de la filière lait, il est important de savoir comment est géré et rémunéré le lait dans les ménages peuls; parce que de cette gestion, dépend la fourniture des marchés du pays en produits laitiers pastoraux.

\section{MATÉRIEL ET MÉTHODES Milieu d'étude}

L'étude a été menée dans la commune de Kalalé. Cette commune a la plus forte concentration en Peuls au Nord du Bénin. Les 
Peuls représentent $68,4 \%$ de la population de cette commune (INSAE, 2003). La commune de Kalalé occupe la seconde place en effectif bovin dans les départements du Borgou et de l'Alibori (Nord-Est du Bénin). Elle est située entre $3^{\circ} 22^{\prime} 54^{\prime \prime}$ de longitude Est et $10^{\circ} 17^{\prime} 24^{\prime \prime}$ de latitude Nord. Cette commune est située en zone soudanienne, avec une alternance de pluies (avril à octobre) et de saison sèche (novembre à mars). La pluviométrie annuelle varie entre 845 et $1322 \mathrm{~mm}$, et suit une distribution unimodale. Le système d'utilisation de la terre est basé sur le sorgho et l'igname, avec une forte concentration en maïs et en coton.

\section{Collecte de données}

La collecte de données a commencé par une enquête exploratoire auprès de 50 chefs de ménages Peuls sélectionnés de façon aléatoire dans trois villages de la commune. Les ménages peuls dans lesquels a été conduite l'étude se sont sédentarisés, et possèdent des troupeaux bovins. Mais en saison sèche, à cause de la rareté du pâturage de bonne qualité, certains membres du ménage partent en transhumance, avec la totalité ou une partie du troupeau bovin et reviennent en saison des pluies.

Les enquêtes se sont déroulées dans seize ménages $(32 \%)$ sélectionnés parmi les cinquante auprès desquels s'est déroulée l'enquête exploratoire. Les données ont été collectées aussi bien en saison sèche, qu'en saison des pluies. Pour s'assurer de la disponibilité du lait pendant ces deux périodes de collecte de données, les seize ménages sélectionnés sont ceux qui ont une bonne production laitière. Car, en saison sèche, la production laitière diminue et la plupart des bouviers préfèrent assurer la croissance des veaux. Dans ces conditions, la quantité de lait traite en saison sèche est faible. Donc, pour mener ce travail, il paraissait intéressant de travailler dans les ménages où le lait serait trait, aussi bien en saison sèche qu'en saison des pluies, pour la consommation humaine. Ces ménages dans lesquels ont été trait, 14 litres de lait par jour se situent dans le dernier quartile.

Un questionnaire a été utilisé pour les enquêtes individuelles, afin de recueillir les données sur les quantités de lait selon l'utilisation qui en est faite, aussi bien au niveau des femmes que des trayeurs. Un guide d'entretien a été utilisé pour conduire des entretiens en focus groupe chez les femmes et les hommes par rapport aux responsabilités des uns et des autres dans la gestion du lait. Les données ont été collectées en mars (période de faible production laitière) et en Juillet (période de forte production laitière) 2014. Les données ont été collectées auprès des membres du ménage qui étaient présents lors des enquêtes.

\section{RÉSULTATS \\ Profil socio-économique des ménages}

Le profil des ménages enquêtés est présenté dans le Tableau 1. Ce tableau montre que les ménages sont de grande taille. Dans chaque ménage, il se côtoie deux à trois générations. A côté des femmes du chef de ménage, il y a également les belles-filles qui sont présentes. Dans les ménages peuls, la majorité des membres s'occupent des activités agricoles alors qu'une minorité est impliquée dans les activités d'élevage. Ceux qui s'occupent des animaux représentent en moyenne $39 \%$ de ceux qui s'adonnent aux activités champêtres. Les femmes s'occupent des activités agricoles autant que les hommes.

\section{Organisation autour de la gestion du capital lait}

En saison des pluies, lorsque le troupeau bovin revient de transhumance, deux cas de figures se présentent, selon l'endroit où reste le troupeau:

Le troupeau est dans un campement, juste à côté de la concession du ménage ou à au plus $5 \mathrm{~km}$;

Le troupeau est situé à plus de $15 \mathrm{~km}$ de la concession du ménage.

Lorsque le troupeau est situé à moins de $5 \mathrm{~km}$ de la concession du ménage, le lait 
est quotidiennement envoyé aux membres du ménage et, ce sont les enfants qui ont la charge d'aller prendre le lait. Mais, lorsque le troupeau bovin est éloigné de la concession (à plus de $15 \mathrm{~km}$ ), et qu'il n'y a pas de vélo pour assurer le transport quotidien, le lait est envoyé aux membres du ménage tous les deux jours. Le transport dans ce cas est le plus souvent assuré par un adolescent. Les autres jours, les membres du ménage achètent dans les troupeaux environnants ou se nourrissent du lait caillé du jour précédent.

Le lait trait dans le troupeau du ménage est majoritairement consommé par les membres du ménage (Tableau 2), mais plus en saison sèche qu'en saison des pluies. Les hommes aussi bien que les femmes peuvent traire le lait. Dans les ménages où il existe plus de femmes, celles-ci traient également le lait. Mais, ce sont les hommes qui dans la majorité des cas traient les vaches. A la fin de la traite, le trayeur garde sa part dans la calebasse qui a servi à la traite. C'est toujours le lait provenant de la dernière vache traite. Le lait trait est divisé en portions égales, au nombre de femmes qui sont dans le troupeau et pour les membres du ménage qui sont dans la grande concession (par exemple, s'il y a trois femmes dans le troupeau, quatre calebasses d'à peu près la même contenance seront présentes lors de la traite: une pour chaque femme et la quatrième pour les autres membres du ménage). Les femmes qui sont dans le troupeau du ménage se réservent une partie du lait obtenu pour leur propre consommation, et une autre pour la vente. Habituellement, ces femmes qui sont dans le troupeau du ménage obtiennent plus de lait que celles qui sont dans la grande concession.

La portion de lait destinée aux autres membres du ménage est également répartie entre les femmes mariées qui sont dans la grande concession. C'est à chaque femme mariée qu'incombe alors la responsabilité de fournir du lait à sa progéniture. Ces femmes mariées qui ne sont pas dans le troupeau du ménage, disposent de juste assez de lait pour leur propre consommation (et celle de leurs enfants). Les vendredis, une partie du lait trait est donnée à toute personne présente lors de la traite. Il se peut que ce soit l'acheteur du lait qui bénéficie de cette dîme.

\section{Rémunération du capital lait}

C'est le dernier fils nouvellement marié accompagné de sa (ses) femme (s) qui amène les animaux en transhumance. Après leur retour de transhumance, c'est toujours ce fils qui s'occupe des animaux. Les femmes qui sont dans le troupeau (les femmes du fils qui a la responsabilité de la conduite du troupeau) détiennent le lait, mais donnent la priorité à la consommation du ménage. La solidarité au sein du ménage ne permet pas à la femme qui est dans le troupeau, de se réserver une grande quantité de lait pour la vente. Mais, c'est toujours les femmes qui restent dans le troupeau, qui vendent le lait issu du troupeau du ménage. Mais des fois, à cause de la grande taille des ménages, et si le ménage ne dispose pas d'un nombre élevé de vaches allaitantes, les femmes qui sont dans le troupeau ne trouvent pas assez de lait, pour la vente et pour la transformation en fromage.

Les femmes peules dont le mari n'a pas la responsabilité du troupeau du ménage ou qui n'a pas de bœufs, profitent du retour de transhumance des troupeaux bovins en saison des pluies, pour s'approvisionner en lait. Lorsqu'il y a des femmes qui veulent acheter du lait, celles qui sont dans le troupeau ne disposent plus d'assez de lait pour fabriquer du fromage. Car une femme peule ne peut pas décider de garder du lait pour elle-même en vue de fabriquer du fromage, alors que d'autres en ont besoin pour leur propre consommation. Au cas où il n'y a pas d'acheteurs, les femmes qui sont dans le troupeau peuvent fabriquer du fromage.

Donc, les femmes qui ne sont pas dans le troupeau du ménage ont plus de possibilités de fabriquer du fromage, comparées à celles qui sont dans le troupeau. La plupart des femmes peules dont les maris n'ont pas de troupeau, produisent régulièrement $\mathrm{du}$ fromage qu'elles vendent le jour du marché. 
Ces femmes achètent du lait dans les troupeaux environnants et vont aussi collecter d'importantes quantités de lait, dans les troupeaux à effectifs élevés (200 têtes) situés assez loin de leurs campements. Les femmes qui fabriquent le fromage le font à tour de rôle. Chacune d'entre elles a un jour où elle part acheter le lait, dans les troupeaux environnants. Elles emportent les bassines et les passoires nécessaires pour fabriquer le fromage sur place, parce qu'elles ne peuvent pas revenir chez elles avec de telles quantités de lait. De ce fait, lorsqu'elles quittent le matin, elles passent toute la journée au lieu d'achat du lait, pour fabriquer et égoutter le fromage avant de revenir à la maison.

\section{Disponibilité des produits laitiers}

En saison sèche, avec le départ de la majorité des troupeaux bovins en transhumance, les femmes peules dont le troupeau n'est pas parti en transhumance préfèrent vendre du lait frais, au lieu de fabriquer du fromage. Le prix du lait est deux fois plus élevé qu'en saison des pluies. Donc le lait frais est le produit le plus disponible dans les villages d'origine des transhumants.

Cependant, dans les zones de transhumance et surtout les centres urbains en périphérie de ces zones, le lait frais est disponible. Mais on rencontre plus de fromage dans ces zones de transhumance que de lait frais. Dans la plupart des cas, c'est le surplus de fromage produit en zone de transhumance qui vient quelques fois alimenter les marchés des communes d'origine des transhumants. Dans la zone de transhumance comme dans les villages d'origine, le prix des produits laitiers en saison sèche est plus élevé qu'en saison des pluies; mais beaucoup plus dans les communes d'origine que dans les zones de transhumance.

Pendant la saison des pluies, cependant, le lait est disponible en abondance; le pâturage de bonne qualité existe et les troupeaux partis en transhumance sont de retour. Le lait et le fromage sont abondants sur tous les marchés et à des prix bas. Le lait est disponible partout (zones de transhumance et villages d'origine des transhumants), et les femmes qui sont dans les troupeaux ont la possibilité de fabriquer du fromage. Mais, à condition que la quantité de lait dont elles disposent ne soit pas achetée par d'autres femmes peules.

Tableau 1 : Profil socio- démographique des ménages.

\begin{tabular}{|c|c|c|c|c|c|c|c|}
\hline & \multirow[t]{2}{*}{ Moyenne } & \multirow[t]{2}{*}{ Mode } & \multirow[t]{2}{*}{ Minimum } & \multirow[t]{2}{*}{ Maximum } & \multicolumn{3}{|c|}{ Quartiles } \\
\hline & & & & & 25 & 50 & 75 \\
\hline Femmes du chef de ménage & 3 & 2 & 2 & 6 & 2 & 3 & 4 \\
\hline Belles-filles & 4 & $0^{\mathrm{a}}$ & 0 & 12 & 1 & 3 & 8 \\
\hline Taille du ménage & 32 & 15 & 9 & 67 & 15 & 30 & 47 \\
\hline $\begin{array}{l}\text { Hommes impliqués dans les } \\
\text { activités d'élevage }\end{array}$ & 3 & $2^{\mathrm{a}}$ & 1 & 5 & 2 & 4 & 5 \\
\hline $\begin{array}{l}\text { Femmes impliquées dans les } \\
\text { activités d'élevage }\end{array}$ & 1 & 0 & 0 & 3 & 0 & 1 & 2 \\
\hline $\begin{array}{l}\text { Hommes impliqués dans les } \\
\text { activités champêtres }\end{array}$ & 8 & $5^{\mathrm{a}}$ & 3 & 17 & 4 & 7 & 10 \\
\hline $\begin{array}{l}\text { Femmes impliquées dans les } \\
\text { activités champêtres }\end{array}$ & 7 & $0^{\mathrm{a}}$ & 0 & 22 & 2 & 6 & 10 \\
\hline
\end{tabular}


Tableau 2 : Gestion du lait dans les ménages peuls selon la saison (en \%).

\begin{tabular}{lcccccc}
\hline & \multicolumn{3}{c}{ Saison sèche } & \multicolumn{3}{c}{ Saison des pluies } \\
\hline & Consommé & Vendu & Donné $^{\mathbf{1}}$ & Consommé & Vendu & Donné \\
\hline Proportion de lait & 78 & 21 & 1 & 58 & 38 & 4 \\
\hline $\begin{array}{l}{ }^{1} \text { : la proportion de lait que les ménages peuls offrent quotidiennement à toute personne étrangère ou à ceux qui sont dans le } \\
\text { besoin. }\end{array}$
\end{tabular}

\section{DISCUSSION}

Organisation autour de la gestion du capital lait

L'organisation mise en place pour la gestion du capital lait permet d'utiliser de façon optimale les ressources en main d'œuvre disponible mais également de faire bénéficier tous les membres du ménage de ce produit issu de l'élevage bovin.

En effet, la fréquence d'envoi du lait aux autres membres du ménage selon le lieu de campement du troupeau participe d'une gestion efficience des ressources en main d'œuvre disponible au sein du ménage. Pendant cette période de pluies (car nous n'avons pas suivi les troupeaux en transhumance), les activités champêtres occupent une grande place dans les activités des ménages peuls. La main d'œuvre disponible est prioritairement affectée aux activités champêtres. Pour les troupeaux proches des concessions, le temps que les enfants (surtout filles) prennent pour aller chercher le lait ne joue pas sur le rendement du travail dans les champs et très souvent, la partie du travail qui leur est réservée peut être réalisée un peu plus tard. Cependant, l'adolescent est déjà assez sollicité par les activités des champs pour qu'un ménage se permette de perdre un temps précieux à envoyer du lait alors que certaines tâches rudes doivent s'accomplir avant que le soleil ne soit au zénith. Cette attitude fait aussi ressortir que le rôle de l'homme ne se limite qu'à la traite du lait et que c'est à la femme qu'incombe la responsabilité d'assurer la disponibilité du lait aussi bien à la maison que sur les marchés. La femme dans son rôle de rendre le lait disponible donne la priorité à la consommation du ménage et surtout à celle des enfants; ce qui fait surtout du lait un produit de subsistance.

L'organisation autour du lait est genre sensible. La traite du lait par l'homme lui permet d'assurer un meilleur arbitrage entre la consommation du lait par le veau et celle des humains. Cette position occupée par l'homme lui permet de ce fait de garantir la reproduction du troupeau, donc sa pérennité. La femme, quant à elle, par son rôle dans la distribution du lait aux membres du ménage, permet la reproduction de la force de travail en assurant une bonne partie des besoins en protéines de la famille. Le droit conféré aux femmes qui sont dans le troupeau de gérer le lait issu du troupeau familial traduit un souci d'équité au sein du ménage. Car c'est elles qui s'occupent des autres tâches du troupeau mis à part la conduite au pâturage et la traite. Donc, dans les systèmes de production laitière des petits producteurs comme chez les Peuls, la femme joue un rôle primordial dans le contrôle et la gestion du lait et des produits laitiers.

La sensibilité au genre dans l'organisation autour du lait apparaît également dans les constats faits par Iyayi et al. (2003) avec les agro-pasteurs Nigérians, et qui rapportèrent que la conduite du troupeau et la traite sont des activités exclusivement 
masculines, alors que la transformation et la vente du lait sont majoritairement réservées aux femmes, dans les centres périurbains du Sud-Ouest du pays. Pour certains auteurs, le contrôle de la traite par les hommes spécialement dans les sociétés pastorales comme les Maasai du Kenya et les Peuls du Nigéria est une façon d'assurer une croissance raisonnable au veau qui est en compétition avec la quantité de lait réservé à la consommation humaine. Dans les sociétés Maasai, comme le souligne Grandin (1988), ce sont les femmes qui traient le lait, mais, les hommes anxieux du fait que le veau puisse avoir assez de lait pour sa survie, tentent de contrôler la traite et font des reproches aux femmes qu'ils croient en train de prendre trop de lait de leurs veaux pour leurs enfants. Pour des raisons similaires, les femmes peules du Nigéria ne traient pas les vaches parce qu'on pense qu'elles prendront trop de lait des veaux (Waters-Bayer, 1985). De plus, cette position des hommes se traduit par le fait que, dans les systèmes d'élevage traditionnels, le défi est de minimiser les mortalités et d'accroître la productivité du cheptel en lait et en viande; mais aussi de maintenir la quantité de lait trait pour la consommation humaine (de'Besi et Thieme, 2013).

Les deux sexes sont impliqués dans la gestion du capital lait mais ce sont plus les femmes peules qui dominent dans l'organisation de la gestion du lait trait. Cette gestion fait ressortir que le lait demeure un produit de subsistance. Son mode de gestion permet de pérenniser le troupeau, de contribuer à la reproduction de la force de travail mais aussi par souci d'équité, de rémunérer le travail des femmes qui s'occupent de l'entretien du troupeau du ménage.

\section{Rémunération du capital lait}

Le lait est l'un des produits les plus importants issus de l'élevage bovin, dans les ménages peuls. Mis à part le fort taux de consommation de ce produit qui procure les protéines aux membres du ménage, la vente du lait contribue à répondre aux autres besoins du ménage.

Les produits laitiers fournissent de l'argent aux femmes dans les ménages peuls. Le fait que ce soit les femmes qui restent dans le troupeau qui bénéficient du lait trait du troupeau participe de la rémunération de leurs efforts de travail dans le troupeau. Cette rémunération leur permet de mieux prendre soin des animaux en compagnie de leurs maris. Mais, le fait que dans la gestion du lait, la consommation des membres du ménage soit privilégiée, expose souvent les femmes qui sont dans les troupeaux à ne pas trouver une grande quantité de lait pour la vente. Si en saison sèche, seules les femmes qui sont dans les troupeaux peuvent bénéficier de la vente du lait, en saison des pluies, beaucoup plus de femmes tirent profit de la vente du lait et surtout du fromage.

En effet, par la position qu'elles occupent, et la quantité additionnelle (la femme qui est dans le troupeau doit aussi participer aux activités champêtres) de travail qui leur revient dans l'entretien du troupeau, les femmes qui restent dans le troupeau vendent souvent du lait frais trait dans le troupeau. Les autres femmes mariées du ménage qui ne sont pas dans le troupeau, quant à elles par l'achat et la transformation du lait dans les autres troupeaux permettent à la valeur ajoutée ainsi créée de rapporter un supplément d'argent aux autres femmes au sein du ménage. Donc cette rémunération du lait est aussi un élément de la pérennisation de l'élevage bovin au sein de la communauté car la distribution de la richesse se fait en grande partie au sein de la communauté, même s'il existe aussi, quelquefois, un intermédiaire non peul entre la femme peule productrice de fromage et le consommateur final.

En Ouganda, la priorité donnée à la consommation du lait dans les ménages de pasteurs explique l'importance de ce produit 
dans les profits courants (Alary et al., 2007). Dans la présente étude, l'importance du lait ressort également dans le fait que les produits laitiers procurent des revenus plus réguliers (revenus journaliers) dans le ménage, comparés à la vente des bovins sur pied et des autres animaux qui est occasionnelle. Selon Lanyasunya et al. (2006), la production laitière est donc la principale raison pour laquelle les bovins sont élevés; et le lait est vendu pour augmenter les revenus des ménages (Olafadehan et Adewumi, 2010).

Dans les sociétés peules, le lait est la propriété de la femme (Kuhn, 1997). Dans la plupart des cas, c'est la femme qui décide de la quantité de lait qu'elle mettra sur le marché, et comment utiliser le revenu issu de la vente du lait au niveau du ménage (FAO, 2005). La transformation du lait et sa vente procure de l'argent aux femmes et contribue à leur autonomie. Ces femmes sont, de ce fait, capables d'acheter ce dont elles ont besoin, s'occuper de leurs enfants et contribuer aux dépenses du ménage. Pour Brockington (2001), la vente du lait est un moyen par lequel les femmes peuvent gagner de l'argent par elles-mêmes. Selon Buhl et Homewood (2000), la commercialisation du lait fournit une opportunité aux femmes de contrôler leur propre argent, lorsque les hommes contrôlent les majeures sources de revenus. La vente du lait contribue aussi à générer les revenus qui peuvent être utilisés dans l'achat des intrants agricoles, incluant les laiteries, et de satisfaire les autres besoins en argent (Somda et al., 2005). Comme le mentionnent encore Somda et al. (2005), le lait n'est pas seulement source de revenus pour les femmes, mais aussi pour tout le ménage. Chez les petits producteurs laitiers du Nigéria et du Kenya, comme dans le cas de cette étude, le lait est produit initialement pour la consommation de la famille, mais aussi pour la vente en vue de financer des urgences d'argent et pour le statut social (Bebe et al., 2003; Iyayi et al., 2003).
En somme, la rémunération du lait et la distribution de la richesse qu'elle induit au sein de la communauté peule en fait un produit très important de l'élevage bovin. Cette importance du lait à travers la communauté peule ressort également à travers l'autoconsommation d'une grande portion au sein des ménages peuls.

\section{Disponibilité des produits laitiers}

La disponibilité des produits laitiers sur les marchés dans le temps et dans l'espace est due aux aléas climatiques mais également aux conséquences de celui-ci sur les pratiques d'élevage. Du fait de la mobilité (transhumance en saison sèche) des troupeaux dans l'année, la disponibilité des produits laitiers varie largement.

L'offre et de la demande du lait, comme de tout produit, conditionne la disponibilité des produits laitiers aussi bien en saison sèche qu'en saison des pluies. En saison sèche, la quantité de lait trait par vache est la plus faible. En plus de cela, la majorité des troupeaux vont en transhumance. Dans cette période de rareté du pâturage et de perte de sa valeur nutritive, la disponibilité du lait s'en trouve affectée dans les villages d'origine des transhumants puisque la demande du lait frais y est supérieure à l'offre. Donc, dans cette période, le lait frais est le produit le plus disponible dans les villages car, d'après les constats faits sur le terrain, la demande existe et n'est pas satisfaite et les femmes peules ne se gênent pas pour ajouter de la valeur au lait (fabrication $\mathrm{du}$ fromage) car les recettes qu'elles tirent de la vente du lait frais sont supérieures à celles qui leur procurent le fromage (1500 francs CFA pour 5 litres de lait alors que le fromage qu'elle peuvent fabriquer à partir de cette quantité de lait sera vendue à peine à 1000 francs CFA). C'est ce qui explique que le fromage soit rare sur les marchés des villages et communes d'origine des transhumants. 
Mais, dans les zones de transhumance, les femmes ont la possibilité de fabriquer du fromage et de le vendre aussi bien que le lait frais. En effet, le nombre de personnes qui suivent le troupeau en transhumance est réduit, donc l'autoconsommation du lait est faible. Ce qui permet aux femmes qui suivent le troupeau en transhumance de disposer d'une quantité suffisante de lait pour la vente. Dans ces zones de transhumance, il y a plusieurs troupeaux; ce qui augmente l'offre de lait. Donc, les femmes qui suivent le troupeau ne peuvent souvent pas vendre toute la quantité de lait frais obtenue quotidiennement. Elles sont alors contraintes de fabriquer du fromage (surtout dans les troupeaux à effectifs élevés) afin de le vendre. Si elles en ont la possibilité, elles peuvent vendre du lait frais à d'autres femmes qui transforment le lait.

C'est ce qui explique que dans les zones de transhumance, le lait aussi bien que le fromage soient disponible toute l'année; même si le prix varie avec le temps. En saison des pluies, les troupeaux locaux fournissent des produits laitiers et en saison sèche, les troupeaux transhumants les rendent disponible.

\section{Conclusion}

La gestion du lait au sein des ménages peuls fait intervenir aussi bien les hommes que les femmes. La position des hommes en amont leur permet ainsi d'assurer la pérennité $\mathrm{du}$ troupeau alors que les femmes s'occupent de la distribution du lait et des produits laitiers dans le ménage et sur les marchés. Le lait demeure un produit de subsistance car sa consommation reste la priorité du ménage. Le supplément de lait trait rémunère le travail de la femme qui est dans le troupeau mais également contribue au partage des bénéfices au sein de toute la communauté peule surtout en saison d'abondance du lait. Ceci montre toute l'importance du lait parmi les produits issus de l'élevage bovin. La gestion de l'effectif du troupeau tout au long de l'année, conséquence de la disponibilité fourragère et la priorité donnée à l'autoconsommation du lait influence la disponibilité des produits laitiers sur les marchés.

Pour le succès des interventions de développement dans le secteur laitier, il faut pleinement apprécier les priorités des ménages peuls et prendre en compte aussi bien les hommes que les femmes car même si le lait est important dans la communauté peule, on ne peut le dissocier de la croissance du troupeau car un équilibre est souvent recherché entre le lait et la viande.

\section{CONFLITS D'INTÉRÊT}

Les auteurs déclarent qu'ils n'ont aucun conflit d'intérêt.

\section{CONTRIBUTIONS DES AUTEURS}

L'étude qui figure dans ce manuscrit représente une partie des recherches doctorales de RCT. PL et AA sont respectivement le directeur et le co-directeur de la thèse.

\section{RÉFÉRENCES}

Aïssi VM, Soumanou MM, Bankolé H, Toukourou F, de Souza CA. 2009. Evaluation of hygienic and mycological quality of local cheese marketed in Benin. Aust. J. Basic Appl. Sci., 3(3): 2397-2404.

Alary V, Chalimbaud J, Faye B. 2007. Multiple Determinants of Milk Production in Africa: The Example of the Diversity of Dairy Farming Systems in the Mbarara Area (Uganda). Africa Development, XXXII(2): 156-180.

Bebe BO, Udo HMJ, Rowlands GJ, Thorpea W. 2003. Smallholder dairy systems in the Kenya highlands: breed preferences and breeding practices. Livestock Production Science, 82: 117-127.

Bierschenk T. 1998. Les Peuls dans le Borgou occidental précolonial. In Regards sur le Borgou - Pouvoir et Altérité dans la Région Ouest-Africaine, Boesen E, 
Hardung C, Kuba R (éds). L'Harmattan: Paris; 175-184.

Bierschenk T, Forster R. 2004. L'Organisation Sociale des Peuls dans l'Est de l'Atacora (République du Bénin, communes de Kouandé, Péhonco et Kérou). Working Papers $N^{\circ} 46$. Johannes Gutenberg Universität Mainz, http://www.ifeas.uni-mainz.de/ workingpapers/Peuls.pdf. Accessed on 25/11/12.

Brockington D. 2001.Women's income and the livelihood strategies of dispossessed pastoralists near the Mkomazi Game Reserve, Tanzania. Human Ecology, 29(3): 307-338.

Buhl S, Homewood K. 2000. Gender and production in Fulani Households: Patterns and determinants of milk selling among pastoral and agropastoralist Fulani women in northern Burkina Faso. In Rethinking Pastoralism in Africa: Gender, Culture and the Myth of the Patriarchal Pastoralist, Hodgson D (ed). Ohio University Press: Athens; 207-226.

de'Besi G, Thieme O. 2013. Optimum use of milk in traditionally managed cattle herds in the tropics. Tropical animal health and Production, 45(5): 1079-1086. DOI: 10.1007/s11250-013-0372-9.

FAO 2004. Perspectives à moyen terme pour les produits agricoles. Projections vers l'an 2010. Food and Agriculture Organization of the United Nations: Rome.

FAO 2005. The future of small scale dairying. Livestock Report 2005. Food and Agriculture Organization of the United Nations: Rome, 45-55.

FAO. 2015a. Dairy production and products. Production system. Food and Agriculture Organization of the United Nations: Rome, http://www.fao.org/ agriculture/ dairy-gateway/milkproduction/production-systems/en/\#. VM9UN52G8m0. Accessed 02/02/2015.
FAO. 2015b. Faostat. Food and Agriculture Organization of the United Nations: Rome, http://faostat3.fao.org/browse/ T/TP/F. Accessed 22/12/2015.

Grandin BE. 1988. Wealth and pastoral dairy production: A case study from Maasailand. Human Ecology, 16: 1-21.

INSAE 2003. Troisième Recensement Général de la Population et de l'Habitation. Synthèse des Résultats. Cotonou, INSAE. http://www.insae-bj.org/2012/doc/ recensement/synthese_des_resultats_rgph 3.pdf. Accessed 12/11/2012.

Iyayi EA, Okoruwa VO, Babayemi OJ, Busari AA, Peters OF. 2003. Livestock production pattern of Agropastoralist in peri-urban centres of South- West Nigeria. Nigerian Journal of Animal Production, 30 (1): 87-92.

Jones R. 1998. The ethnic groups of present day Borgu. In Regards sur le Borgou Pouvoir et Altérité dans la Région OuestAfricaine, Boesen E, Hardung C, Kuba R (éds). L'Harmattan: Paris; 71-89.

Kuhn B. 1997. «Kosam walaa ceede walaa!» «Pas de lait, pas d'argent !» L'importance du lait chez les femmes peules du NordBénin. In Trajectoires Peules au Bénin. Six Etudes Anthropologiques, Bierschenk T, Le Meur P-Y (éds). Karthala : Paris; 63-76.

Lanyasunya TP, Wang HR, Mukisira ER, Abdulrazak SA, Ayako WO. 2006. Effect of seasonality on feed availability, quality and herd performance on smallholder farms in Ol-Joro-Orok Location/Nyandarua District, Kenya. Tropical and Subtropical Agroecosystems, 6: 87 - 93.

Majekodunmi AO, Fajinmi A, Dongkum C, Shaw APM, Welburn SC. 2014. Pastoral livelihoods of the Fulani on the Jos Plateau of Nigeria. Pastoralism: Research, Policy and Practice, 4: 20. DOI:10.1186/s13570-014-0020-7 
Olafadehan OA, Adewumi MK. 2010. Livestock management and production system of agropastoralists in the derived savanna of south-west Nigeria. Tropical and Subtropical Agroecosystems, 12: 685 -691 .

Sessou P, Farougou S, Azokpota P, Youssao I, Yehouenou B, Ahounou S, Codjo Koko Sohounhloue D. 2013. Inventaire et analyse des pratiques endogènes de conservation du wagashi, un fromage traditionnel produit au Bénin. Int. J. Biol. Chem. Sci., 7(3): 938-952. DOI : http://dx.doi.org/10.4314/ ijbcs.v7i3.5

Somda J, Kamuanga M, Mendes A, Gomes J. 2004. Caractéristiques socioéconomiques et performances économiques des élevages laitiers en Guinée Bissau : cas des régions de Bafata et Gabu. Socio-economic Research Working Paper No 4, ITC (International
Trypanotolerance Centre), Banjul, The Gambia, $48 \mathrm{p}$.

Somda J, Mulumba Kamuanga M, Tollens E. 2005. Characteristics and economic viability of milk production in the smallholder farming systems in The Gambia. Agricultural Systems, 85: 42-58. DOI:10.1016/j.agsy.2004.07.011

Waters-Bayer A. 1985. Dairying by settled Fulani women in central Nigeria and some implications for dairy development, Pastoral Development Network Paper 20c, Overseas Development Institute, London; $26 \mathrm{p}$.

Youssao AKI, Dahouda M, Attakpa EY, Koutinhouin GB, Ahounou GS, Toleba SS, Balogoun BS. 2013. Diversité des systèmes d'élevages de bovins de race bovine Borgou dans la zone soudanienne du Bénin. Int. J. Biol. Chem. Sci., 7(1): 125-146. DOI : http://dx.doi.org/10. 4314/ijbcs.v7i1i.11. 